Service social

\title{
Le défi du futur
}

\section{Christiane Besson, Allan Brown, Ken Heap, Hyam Samaha-Kahi et Ben Zion Shapiro}

Volume 39, numéro 1, 1990

Le groupe ici et ailleurs

URI : https://id.erudit.org/iderudit/706461ar

DOI : https://doi.org/10.7202/706461ar

Aller au sommaire du numéro

Éditeur(s)

École de service social de l'Université Laval

ISSN

1708-1734 (numérique)

Découvrir la revue

Citer cet article

Besson, C., Brown, A., Heap, K., Samaha-Kahi, H. \& Shapiro, B. Z. (1990). Le défi du futur. Service social, 39(1), 138-150. https://doi.org/10.7202/706461ar

\section{Résumé de l'article}

Les textes qui composent cet article proviennent de communications présentées lors du panel de clôture du $11^{\mathrm{e}}$ symposium sur le service social des groupes. Ce panel avait pour but de partager la vision de cinq spécialistes de différents pays concernant les défis auxquels seront confrontés les travailleurs sociaux dans cette décennie qui commence. d'utilisation que vous pouvez consulter en ligne.

https://apropos.erudit.org/fr/usagers/politique-dutilisation/ 


\section{Le défi du futur}

Les textes qui suivent proviennent de communications présentées lors du panel international qui a clôturé le $11^{\mathrm{e}}$ symposium sur le service social des groupes. Ce panel avait pour but de partager la vision de cinq spécialistes de différents pays concernant les défis auxquels seront confrontés les travailleurs sociaux dans cette nouvelle décennie qui commence.

Nous débutons avec la communication présentée par madame Christiane Besson de Suisse ${ }^{1}$. Nous poursuivons avec les résumés des communications des panellistes suivants : Allan Brown d'Angleterre ${ }^{2}$,

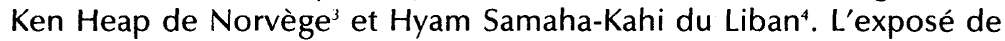
Ben Zion Shapiro du Canada ${ }^{5}$ complète cet article.

\section{Notes}

${ }^{1}$ Cette communication, la seule à avoir été présentée en français, est reproduite ici au complet.

${ }^{2}$ Communication présentée en anglais, résumée et traduite par Lise DarveauFournier et Alice M. Home.

${ }^{3}$ Ken Heap n'a pu se rendre à Montréal en raison de maladie. Le texte anglais de sa communication a été résumé et traduit par Élaine Carey-Bélanger.

${ }^{4}$ Les conditions de guerre au Liban ont empêché madame Samaha-Kahi de participer au panel. Nous présentons le résumé de sa communication.

${ }^{5}$ Communication présentée en anglais, traduite par Lise Darveau-Fournier. 
Christiane Besson, responsable de formation continue pour travailleurs sociaux, Saint-Légier, Suisse.

Car alors te résiste la matière même dont tu dois faire usage, alors que ne résisteront jamais tes inventions sur l'avenir.

(Saint-Exupéry, $1953: 651$ )

Parler des défis du futur, de ce que nous pourrions, devrions être demain, n'est-ce pas évoquer les puissances, les dieux et les démons, conjurer, exorciser, promettre, menacer, jouer les Cassandre... Les prophètes sont pessimistes... et toujours on les suspecte de provoquer les malheurs qu'ils annoncent.

Permettez-moi d'emprunter une image à l'un des merveilleux albums de Rodolphe Toepffer (1975) lorsqu'il nous montre M. Cryptogame disant adieu à l'Europe et s'embarquant pour I'Amérique. M. Cryptogame tourne le dos à sa destination, comme le rameur auquel un savant genevois, $M$. Jean-Claude Courvoisier (1985), consacrait un livre passionnant sur le thème du temps et qu'il intitule précisément : Le Mythe du rameur. Comparant l'homme au rameur poussant son esquif sur l'eau et tournant par conséquent le dos à l'objectif qu'il poursuit, $M$. Courvoisier s'interroge : le rameur peut-il se retourner pour contempler son avenir en face ? La réponse est négative : la vie conduit l'homme sur un chemin dont la direction peut être tracée d'avance, mais jamais connue d'avance, et le sens du voyage apparaît seulement à mesure que, devant lui, à l'arrière du bateau, I'horizon s'élargit peu à peu.

\section{Retrouver le sens de l'aventure : l'aventurier, l'explorateur}

Les structures rigides, les castes antagonistes et jalouses de leurs privilèges, le manque de mobilité, inhibent la créativité et l'innovation.

(Joël de Rosnay, 1983 : 115)

La vie est une aventure. On l'oublie quelque peu aujourd'hui et I'homme cóntemporain est moins un aventurier qu'un touriste, suivant, guide bleu en main, les chemins que d'autres ont explorés pour lui. L'aventurier, c'est "celui qui oublie où mène le chemin ", comme dit Héraclite, celui dont l'attention est toute tournée vers ce qui advient, dans l'instant, celui qui met sa confiance dans l'avenir sans se préoccuper de le deviner d'avance, bref I'homme entièrement présent. 
Au fond, le seul courage qui nous est demandé est de faire face à l'étrange, au merveilleux, à l'inexplicable que nous rencontrons. [...] La peur de l'inexplicable n'a pas seulement appauvri l'existence de l'individu, mais encore les rapports d'homme à homme, elle les a soustraits au fleuve des possibilités infinies, pour les abriter en quelque lieu sûr de la rive. Ce n'est pas seulement à la paresse que les rapports d'homme à homme doivent d'être indiciblement monotones, de se reproduire sans nouveautés : c'est à l'appréhension par l'homme d'un nouveau dont il ne peut prévoir l'issue et qu'il ne se sent pas de taille à affronter. Celui-là seulement qui s'attend à tout, qui n'exclut rien, pas même l'énigme, vivra les rapports $d^{\prime}$ homme à homme comme de la vie, et en même temps ira au bout de sa propre vie.

(Rilke, $1937: 92-94)$

II devient indispensable de retrouver le sens de l'imprévu, le sens d'un accueil inconditionnel du client. Le travailleur social de groupe n'est pas d'abord un fonctionnaire préposé au triage. Les spécialistes ont leur place, mais sans raisonner en géomètres, cartographes ou patrons d'agences de voyage. Pour nos groupes, je voudrais des poètes, des philosophes, des hommes et des femmes des sciences expérimentales, des botanistes, des alchimistes.

La science moderne a mis au cœur de l'homme l'ambition de prévoir le cours des choses et de le modifier. Ce qu'il a perdu du même coup, c'est la capacité d'accepter l'incertitude, l'imprévisibilité de l'existence. II faut cesser d'établir des projets, d'attendre, pour devenir disponible à l'imprévu.

\section{Augmenter la capacité à rétablir des liens : le médiateur}

Cloisonnements et blocages stérilisent les germes du changement, entravent les courants d'innovation. [...] On pourrait imaginer un [...] lubrifiant sous la forme du "tiers secteur», constitué par les mouvements associatifs et les fondations. Il met au service de I'intérêt général volontariat, bénévolat et mécénat.

(Joël de Rosnay, 1983 : 124-125)

II faut cesser d'opposer la compétence et la vocation, les questions matérielles et spirituelles, le progrès technique et le progrès social. L'histoire nous a conduits à opposer, de façon un peu simpliste il est vrai, les professionnels d'aujourd'hui aux bénévoles $d^{\prime}$ autrefois. En réalité si on y réfléchit, le professionnalisme s'oppose à l'amateurisme tandis que le bénévolat s'oppose au salariat. II y a là . encore une opposition qu'il faudra dépasser. L'avenir permettra de réconcilier le salarié et le bénévole, l'amateur et le professionnel. Le travailleur social de groupe est fort bien placé pour œuvrer à cette réconciliation. II peut inventer, grâce à la vie de groupe, quelque chose de différent qui consistera pour lui à ne pas agir seulement en 
fonction de son statut de salarié, mais de faire aussi certaines choses parce qu'il le veut bien, ce qui est proprement le bénévolat, à faire aussi les choses de son métier en amateur, c'est-à-dire parce qu'il aime son métier. Le groupe, on le sait bien, remet en cause le statut de son responsable; il faudra de plus en plus résister à l'emprise sclérosante du statut. Car le professionnalisme implique la mise en place d'un statut, c'est-à-dire le fait d'être reconnu, correctement rémunéré, et de bénéficier de conditions de travail raisonnables, toutes choses maintenant acquises.

Tant que la société reste organisée sous une forme "pyramidale " avec ses niveaux hiérarchiques bien définis reposant sur une spécialisation étroite des tâches, l'exercice du pouvoir répond aux schémas traditionnels. Il est plus aisé et plus efficace que dans un réseau multiforme. La tête la plus haut placée est censée penser pour toutes les autres. Or l'avènement du " réseau pensant " fait éclater de telles structures, redistribue les pouvoirs. Ce sont donc de nouvelles formes d'exercice et de distribution du pouvoir qu'il faut inventer.

(Joël de Rosnay, 1983 : 151)

Nous devons également assumer la tension entre les deux solidarités dont notre métier est tributaire, celle qui nous unit aux marginaux et celle qui nous unit au monde de la normalité. Le groupe me paraît là encore être un espace transitionnel (au sens de Winnicott, 1951) où peuvent se jouer et se négocier ces tensions.

C'est Christophe le passeur qu'il nous faut évoquer, constamment en transit entre les deux rives de la normalité et de la marginalité (Baudouin, 1987). Saint Christophe ne pourrait faire son métier s'il n'acceptait de se mouiller les pieds. II faut résister à la tentation d'un travail social « en blouse blanche et stylo feutre » auquel succombent certains professionnels au nom du professionnalisme.

\section{Redécouvrir la valeur de l'artisanat : I'artisan}

II s'agit de revoir notre conception des relations entre la pensée et l'action. Face à tout problème nouveau ou plutôt face à un problème dont on prend conscience et de façon nouvelle, il est courant que l'on crée une commission formée de professionnels présumés compétents, en la chargeant de définir une politique, si possible globale, pour diriger l'action de ceux qui doivent agir dans le domaine considéré.

Je crois que l'action subit la loi de certains principes dont le premier est qu'elle n'est pas tout incluse dans la pensée qui la précède. II faut au contraire que l'action soit entreprise et déjà mise en œuvre pour développer sa vie propre par tâtonnements, essais et erreurs. C'est tout particulièrement vrai en travail social de groupe. 
À mes yeux, le travail social de groupe est un art, il fait partie des métiers artisanaux et en possède les caractéristiques dont la principale est que la pensée et l'action y sont étroitement unies et exercent l'une sur l'autre une influence réciproque permanente. Un effet regrettable produit par la spécialisation réside dans la séparation de la pensée et de l'action, d'abord en isolant les secteurs d'activités les uns des autres et en multipliant les échelons de la hiérarchie.

II doit être possible, et le travail de groupe va dans ce sens, de réaliser des conditions organisationnelles où la pensée et l'action soient regroupées où les compétences de décision et la responsabilité soient assumées par les personnes les plus proches, voire appartenant aux milieux concernés. II faut retirer au pouvoir celui de définir l'action à l'avance et de façon globalisante. II faut exiger de lui qu'il l'exerce comme un art, non comme une science exacte.

\section{Lutter contre la pesanteur institutionnelle : le funambule, l'acrobate}

La contamination par la pesanteur institutionnelle guette le travailleur social. La mélancolie s'institutionnalise et une certaine léthargie risque de s'installer.

Mieux se connaître, évaluer ses potentialités et ses limites, c'est aussi mieux comprendre l'autre. Chacun apparaît ainsi comme un nœud dans un réseau de relations, d'informations et d'échanges. La constitution de tels réseaux favorise, en retour, la personnalisation et la responsabilisation de chaque individu, tout en faisant ressortir son rôle unique dans une communauté d'intérêts ou de valeurs partagées.

(Joël de Rosnay, 1983 : 16)

De cette mélancolie, on ne sortira que par des secousses imposées à soi-même, par le refus des routines inutiles et peut-être aussi par la réceptivité à la grâce qui, venant d'ailleurs, nous délie de toutes les pesanteurs. " [...] l'avenir n'est autre chose pour moi que ce qui ne m'est pas immédiatement donné »(Weil, 1988 : 149). L'être à qui tout est donné et à qui l'exigence de l'action n'est pas imposée par les circonstances est donc privé de toute existence dans le temps. $C^{\prime}$ est la situation que, par notre activisme débordant, nous finissons par créer pour certains clients. L'essentiel de notre rôle comme travailleur social de groupe pourrait bien consister de plus en plus à apprendre à en faire de moins en moins. Ce que je cesse de faire crée les conditions pour que mon vis-à-vis, dans le cadre d'un groupe, sorte de sa passivité et entreprenne de faire lui-même. C'est à cette condition que l'individu invente sa liberté et se crée un avenir par l'action qui le constitue comme un être en devenir, comme être dans la mouvance du temps. 


\section{Préparer l'avenir en vivant au présent}

Préparer l'avenir, c'est agir au présent, vivre au jour le jour... Lutter au jour le jour, comme Jacob avec l'ange, pour que triomphe l'essentiel, soit les trois aspects fondamentaux de l'être humain : l'âme, le corps et l'esprit. Sur le plan de l'esprit, il s'agit d'un combat avec l'ange de la science, qui voudrait imposer le tribut du savoir. Là se réconcilient compétence et vocation. Sur le plan du corps, c'est le combat avec l'ange de la pesanteur matérielle et organisationnelle, qui voudrait imposer le tribut de la bureaucratie. Là se réconcilient le bien-être et le confort avec la présence et la disponibilité. Sur le plan de l'âme, c'est le combat avec l'ange de la douleur et de l'échec, qui voudrait imposer le tribut de la dépression. Là enfin, l'individu est appelé à se réconcilier avec sa propre souffrance.

Préparer l'avenir en vivant au présent, ce n'est pas s'arrêter pour regarder son avenir en face. C'est contempler, mais d'une autre façon. À la manière de Michel-Ange qui, se promenant avec des amis, s'arrêta soudain, longuement, devant un bloc de marbre; pressé par eux de les rejoindre, il leur dit : "il y a un ange dans cette pierre..." .

Lorsque nous créons des groupes en travail social, si nous faisons bien notre travail, il en résulte des effets tels que des liens d'amitié, des actes d'entraide, une série d'expériences d'interactions dans un milieu protégé qui permettront de nouvelles actions dans d'autres lieux, en bref, une mobilisation. Ainsi naissent des réseaux secondaires informels.

[...] les réseaux constituent [...] une forme alternative d'organisation de la société. Un relai pour la société industrielle héritée du $\mathrm{XIX}^{\mathrm{e}}$ siècle. Une nouvelle phase à laquelle nous devons nous préparer pour mieux nous y adapter. [...] Un réseau est [...] une réponse adaptative à une nécessité de développement rapide à partir d'entités éphémères.

(Joël de Rosnay, 1983 : 185)

Le futur a déjà commencé !

\section{Références}

Baudouin, Charles (1987). Christophe le passeur. Paris : Le Courrier du Livre. COURVOISIER, Jean-Claude (1985). Le mythe du rameur. Lausanne : Éditions de l'Aire.

De Rosnay, Joël (1983). Les chemins de la vie. Paris : Seuil.

RILKE, Rainer-Maria (1937). Lettres à un jeune poète. Paris : Grasset.

SAINT-EXUPÉRY, Antoine de (1953). Citadelle, dans Oeuvres, Bibliothèque de la Pléiade. Paris : Gallimard. 
TOEPFFER, Rodolphe (1975). Monsieur Cryptogame s'embarque pour l'Amérique. Lausanne. (Imprimé en France pour La Guilde du Livre).

WeIL, Simone (1988). Oeuvres complètes. Tome 1 : Premiers écrits philosophiques. Paris : Gallimard.

WINNICOTT, D.W. (1951). "Transitional Objects and Transitional Phenomena ", dans Collected Papers. Through Pediatrics to Psycho-Analysis. London : Tavistock Publications. Concepts revus par l'auteur (Winnicott, 1975), dans Jeu et réalité. Paris : Gallimard.

Allan Brown, directeur et professeur, Département de service social, Université de Bristol, Angleterre. Coéditeur de la revue Groupwork.

Les travailleurs sociaux de groupe à travers le monde ont en commun la conviction que le groupe peut conduire au changement, à l'enrichissement et à l'augmentation du pouvoir des individus, des groupes et des communautés, quelles que soient leurs conditions de vie et de travail. Le travail de groupe en Grande-Bretagne doit être vu dans un contexte de thatchérisme, philosophie diamétralement opposée aux valeurs de responsabilité partagée et de collectivisation des solutions, aux problèmes d'entraide et de partage de pouvoir qui caractérisent le service social des groupes. Elle met en valeur l'idéologie d'individualisme, de profit, de "tiens-toi debout par tes propres ressources", prônée par un premier ministre qui a affirmé publiquement : " la société n'existe pas en soi, il y a des individus, hommes et femmes, et il y a des familles ". Les travailleurs sociaux voient les politiques sociales de madame Thatcher comme causes d'oppression (surtout des femmes, minorités, chômeurs et handicapés) et de pauvreté, de désespoir et, pire, de perte d'estime de soi.

Les approches de groupe reflètent cette polarisation et particulièrement le contraste entre le modèle de changement de comportement et celui d'action sociale. Le premier insiste sur la fonction de contrôle du travailleur social, attachant peu d'importance au contexte social; le deuxième met l'accent sur l'atteinte de buts externes au groupe, insistant peu sur le processus du groupe et la responsabilité individuelle. L'intérêt et la préoccupation envers l'ethnicité et le sexe, en relation avec la théorie et l'impact sur la pratique, constituent un autre courant important. II en résulte une augmentation du nombre de groupes spécifiquement en rapport avec la race et le sexe, de même qu'une reconnaissance d'une respon- 
sabilité particulière des travailleurs sociaux qui interviennent auprès de groupes mixtes : ils doivent aider leurs groupes à éviter la reproduction des stéréotypes sociaux.

Ces courants de pensée ont plusieurs effets sur la pratique du service social des groupes. Premièrement, nous devons éviter la division classique entre groupes de changement personnel et groupes de changement social. Nous ne pouvons offrir un service complet aux membres des groupes sans tenir compte de l'influence des facteurs individuels, de groupe et environnementaux.

En second lieu, nous devons rester vigilants lorsque, dans chacun des groupes où nous travaillons, nous utilisons des stéréotypes sociaux. Il en est de même dans notre travail, au sein de notre famille, de nos groupes de loisirs, ainsi que dans notre rôle professionnel auprès de tout groupe de clients.

Troisièmement, l'absence d'une association de travailleurs sociaux en Angleterre entraîne l'isolement des intervenants; il en résulte un impact direct sur la qualité de la pratique de groupe et sur le développement du travail de groupe comme méthode importante d'intervention. Malgré les efforts déjà tentés pour créer un réseau et en dépit de l'intérêt des travailleurs sociaux de groupe, nous rencontrons des dilemmes semblables à ceux qu'a connus I'AASWG (Association for the Advancement of Social Work with Groups) à ses débuts, au sujet de la forme et du type d'organisation appropriés.

Finalement, après une période de dépendance excessive envers les publications nord-américaines, le mouvement est en train de s'inverser. La revue Groupwork, offrant un numéro spécial européen édité par Ken Heap, contribue à ce changement. II ne faudrait toutefois pas négliger de tirer profit des connaissances provenant des autres pays. Les nouveaux réseaux établis avec nos collègues européens devraient nous aider à renforcer nos relations avec les travailleurs sociaux de groupe de l'Amérique du Nord et à en établir d'autres avec nos collègues du reste du monde.

Ken Heap, professeur,

École luthérienne de service social, Oslo, Norvège.

On remarque, en Norvège et dans les pays scandinaves, une participation accrue des citoyens, une conscience plus développée des droits et responsabilités, un scepticisme plus grand envers la 
validité et la pertinence des solutions politiques. La méthode de groupe reflète déjà les tendances, besoins et problèmes prévisibles pour les vingt-cinq ans à venir. Elle se caractérise par une diversité, à savoir son application dans presque tous les milieux de dispensation des services de santé et des services sociaux, et par une créativité, soit des techniques innovatrices et expérimentales pour faire face aux problématiques nouvelles (abus sexuels, conflits culturels, etc.). De tels développements reflètent la réponse du service social des groupes, sur le plan international, aux problèmes sociétaux. Ce don $d^{\prime}$ inventer et de saisir les opportunités est caractéristique du travail social de groupe dans les pays scandinaves. Nous pouvons prédire que les problèmes suivants vont aller en s'intensifiant : crise économique, chômage, vieillissement de la population, émigration, immigration, réfugiés, déplacements. L'investissement du travail social de groupe dans toutes ces problématiques va aussi augmenter.

II faut toutefois souligner quelques particularités de l'intervention de groupe en Scandinavie. D'une part, on y a toujours associé les racines et la tradition du service social des groupes au mouvement des "settlements " de Jane Adams. D'autre part, on s'est inspiré fortement de la psychothérapie de groupe. Ce phénomène découle des limites de l'approche individuelle, lesquelles ont incité les travailleurs sociaux à développer d'autres compétences. Cette utilisation très habile des groupes a été, jusqu'à maintenant, surtout thérapeutique, pragmatique et dirigée vers la solution de problèmes.

Les besoins du futur se feront surtout sentir au niveau des problèmes sociétaux, de communication et d'interaction, ce qui exigera :

- une réorganisation des programmes d'études;

- un accent, durant la formation, sur le développement de la " conscientisation »;

- des programmes de formation continue, de suivi ou de consultation professionnelle, et de réorientation;

- une clarification des concepts de réseaux, de groupes d'entraide;

- une remotivation des intervenants à travailler dans le sens de l'augmentation des ressources, de l'autonomie, de la qualité de vie et de l'interdépendance. 
Hyam Samaha-Kahi, directrice et professeure, École libanaise de formation sociale, Université SaintJoseph, Beyrouth, Liban.

Au Liban, les méthodes de groupe correspondent parfaitement au contexte socioculturel. Elles sont de plus en plus introduites au niveau de l'action (avec les populations déplacées, les veuves, les handicapés de la guerre et leurs parents, etc.) et au niveau de la formation de base et de la formation continue.

Je crois profondément que le groupe est une occasion pour que I'individu ne verse pas dans son individualisme. Malheureusement, les sociétés modernes ont plutôt fait émerger l'individualisme aux dépens de l'individualité de chaque être. J'entends par individualisme la centration sur soi-même aux dépens de l'autre et, par individualité, la réalisation de soi et la prise de conscience de ses forces d'ouverture à l'autre dans un rapport d'égalité, de mutualité et d'interdépendance.

Je suis convaincue que le vécu du groupe est un moment des plus favorables qui aide la personne à développer son individualité et à dépasser les grandes idées de liberté et de justice pour les vivre concrètement dans un rapport d'interactivité réelle, dans un contexte où la seule solidarité est souvent un groupe devenant un refuge contre un monde qui semble sans cœur.

L'expérience du Liban ne cesse de nous prouver et de confirmer ces idées.

Ben Zion Shapiro, professeur, Faculté de service social, Université de Toronto.

Nos outils les plus précieux pour regarder vers l'avenir sont notre sens de l'aventure et notre capacité d'examiner le passé. En ce qui concerne l'avenir, nous ne pouvons formuler que des questions, non des réponses. Le défi consiste à trouver les bonnes questions. Ces dernières s'inspirent de nos craintes et inquiétudes ainsi que de nos espoirs de changement; mais elles se modèlent sur nos façons habituelles de penser, lesquelles sont influencées par le temps et le lieu, la culture et l'expérience. 
Le fait de considérer le service social des groupes dans une perspective internationale devrait nous aider à reconnaître que nous n'avons pas tous les mêmes interrogations. Ma propre pratique et mon expérience d'enseignement au Canada, en Israël et aux ÉtatsUnis, de même que mes visites dans plusieurs régions de l'Europe de I'Ouest comme de l'Europe de l'Est tendent à le confirmer. Mais je crois que nous pouvons partager certaines catégories de questions qui reflètent une convergence de préoccupations, entre autres vers le politique, le technologique et le moral.

La plupart de nos questions courantes sont politiques; elles concernent la répartition du pouvoir entre les groupes dans une société et entre les sociétés. Les travailleurs sociaux de groupe observent ces enjeux dans leurs propres groupes, entre les membres de leurs groupes et entre les groupes et la communauté.

Comme les gens entrent de plus en plus en contact les uns avec les autres à travers les migrations, et certainement par les médias électroniques, nous partageons et comparons nos situations en ce qui concerne la race, l'ethnicité, la classe sociale, le sexe, la souveraineté politique. Nous avons de la difficulté à accepter nos différences telles qu'elles sont; nous souhaitons avoir davantage le pouvoir de choisir nos différences. Les revendications concurrentes de diverses communautés pour obtenir des droits et libertés, de même que des avantages économiques et sociaux, se reflètent dans notre vie quotidienne concrète. Nous utilisons de plus en plus le langage du pouvoir dans nos analyses de situations et dans la formulation d'objectifs. L'accroissement du pouvoir des individus et des groupes fait partie de l'agenda et dépasse de loin les relations entre individus; cela entraîne des implications sociales et structurelles fondamentales et soulève des questions au sujet des hypothèses que nous faisons et des structures que nous acceptons et maintenons.

Notre questionnement implique de plus en plus la recherche d'une meilleure technologie dans les domaines des relations humaines et de l'aide interpersonnelle et, de façon plus générale, dans celui des politiques de bien-être social. Nous sommes de plus en plus fascinés par la méthode et la modalité, par l'invention et l'innovation. Même la réaction à la technologie et à l'ingénierie humaine n'échappe pas aux styles et rituels alternatifs. À mesure que nous nous familiarisons avec le langage du pouvoir, nous devenons des experts de la technique. Nous nous posons d'importantes questions sur l'efficacité de nos interventions et sur le développement des sciences appliquées. Mais nous ne devons pas oublier d'examiner, de façon critique, les prémisses de base qui sous-tendent nos structures de pensée et, bien sûr, nos constructions de la réalité; que voyons-nous et comprenons-nous et que négligeons-nous de regarder et de 
comprendre au sujet des personnes dans les groupes et au sujet de notre réalité sociale dans son ensemble? Nous oublions souvent d'essayer de découvrir les changements de paradigme, les défis parfois subtils qui permettent d'aller au-delà de la pensée et de l'analyse conventionnelles et qui peuvent contenir en eux-mêmes les germes de futures révolutions dans la pensée et les pratiques. C'est pourtant ce vers quoi nous devons tendre si nous voulons faire face au défi du futur et du changement.

Le troisième type de questions que je soulève concerne le moral; nous référons ici au langage de la responsabilité. Nous nous intéressons de plus en plus à l'étendue et aux limites de la responsabilité publique, tant dans le secteur gouvernemental que volontaire, en rapport avec les initiatives commerciales et privées d'une part, et avec les réseaux et processus informels d'autre part. Nous considérons la formalisation de l'entraide et l'organisation des systèmes de soutien comme l'expression de saines initiatives issues de la base, mais aussi comme l'abdication par le gouvernement de ses responsabilités. À l'échelle internationale, nous observons une augmentation des liens intercorporatifs entre les multinationales et un désengagement face aux communautés locales. Mais nous sommes aussi témoins de préoccupations transnationales au sujet du développement humain et des conséquences humaines des dommages à l'environnement. Nous nous préoccupons des implications éthiques et morales des conflits politiques, de la technologie médicale et génétique; nous nous préoccupons aussi des questions relatives aux personnes qui assument diverses responsabilités dans ces domaines. Ces préoccupations se retrouvent aussi dans nos groupes et dans les rapports inter-groupes. Le rôle et la responsabilité du travailleur social de groupe, en tant que professionnel détenteur de pouvoir et de technologie, présente une dimension morale importante et soulève d'épineuses questions.

Dans l'examen de ces trois catégories de questions, il serait bon de nous rappeler que les pionniers du service social des groupes ont présenté un paradigme des groupes que nous oublions parfois. Nous pouvons nous appuyer sur le fait qu'ils considéraient les groupes comme fondements de la société. En d'autres temps et lieux, le groupe en tant qu'entité sociale a été vu comme instrument pour assurer la continuité sociale, comme moyen de contrôle social, comme possibilité de prendre un recul face à la société, comme protection contre des normes sociales subversives, comme tampon contre le stress, comme un lieu d'aide mutuelle ou comme un véhicule de changement social. Même dans le cadre du service social, le groupe a été perçu parfois comme moyen de réussir un changement personnel et un accroissement de pouvoir personnel, parfois 
comme instrument d'action collective et d'accroissement de pouvoir collectif. Dans certains cas, le groupe a servi d'audience à des processus hautement individualisés.

Selon moi, le défi du futur pour le service social des groupes est d'examiner la place des groupes humains dans la réalité contemporaine de nos sociétés respectives, quelquefois très différentes, et de trouver réponse à nos questions politiques, technologiques et morales de façon à prendre en compte des paradigmes de groupe qui peuvent être différents et nouveaux.

Peut-être qu'en agissant ainsi, nous réussirons à éviter les anciennes tendances à exporter et à importer les idées et les pratiques, incluant la théorie de l'intervention de groupe, sans tenir compte de l'expression authentique des différences humaines. D'un certain point de vue, ce fait culturel est aussi un acte politique que l'on peut définir comme une forme de colonisation, fondé sur la prémisse que les conceptions de l'aide devraient provenir d'une société économiquement forte vers une société dépendante au plan économique, d'une société développée au plan scientifique vers une autre qui ne possède pas les mêmes traditions académiques. Devrions-nous continuer à agir ainsi ? En fait, le pourrions-nous même si nous le voulions?

Le " village global » de McLuhan ressemble davantage à un village global composé de villages globaux, chacun se partageant, dans une combinaison unique, des parties du tout, tous interdépendants, ne suivant pourtant pas de sentiers uniques. Notre capacité d'apprendre le langage et les habiletés des réseaux sera cruciale. Nous devrons franchir frontières et obstacles pour établir des liens partout où nous le pouvons et pour assurer que les informations et idées se propagent dans plusieurs directions et sans interruption. Le défi du futur devra se transformer en un défi de plusieurs futurs. 